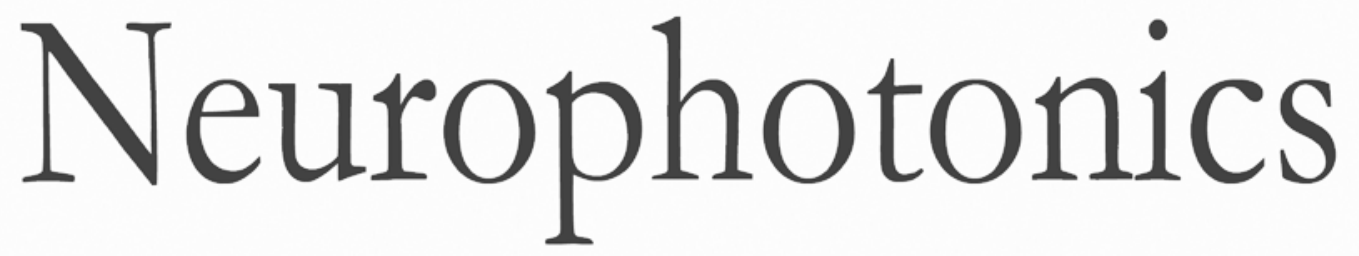

Neurophotonics.SPIEDigitalLibrary.org

\title{
Special Section on Light Microscopy of Connectivity
}

Shaoqun Zeng Timothy H. Murphy

\section{SPIE.}




\section{Special Section on Light Microscopy of Connectivity}

\author{
Shaoqun Zeng \\ Huazhong University of Science and Technology \\ Britton Chance Center for Biomedical Photonics \\ Department of Biomedical Engineering \\ 1037 Luoyu Road, Wuhan, 430074 China \\ E-mail: sqzeng@mail.hust.edu.cn
}

\section{Timothy H. Murphy}

University of British Columbia at Vancouver

Department of Psychiatry

British Columbia V6T 1Z3, Canada

E-mail: thmurphy@mail.ubc.ca
The mammalian brain is a complex system containing billions of neurons, and every neuron forms thousands of connections with other neurons, with information flowing from one neuron to another. Thus the neuronal connection map, a kind of "information highway," plays a key role in understanding how the brain works. However, with hundreds years of development, investigators cannot draw out the complete structure of complex neurons, let alone the full connection map. Knowledge deficiency of brain connectivity is actually a result of lacking proper tools. In recent years, techniques have emerged that raise the potential to address such questions. Diffusion tension imaging-magnetic resonance imaging has provided a coarse map of different brain areas, the so-called "connectome," and launched the Human Connectome Project. Serial electron microscopy has dissected brain tissue to as fine as a nanometer, and thus is able to map each synapse, the connection site between two neurons, and have been extending to large tissue blocks. With the help of molecular labeling, optical microscopic imaging fills the gap between electron microscopy and magnetic resonance imaging, and has been playing an important role in exploring the organization and function of the brain. The Special Section on Light Microscopy of Connectivity shows recent efforts to image the fine organization and function using light microscopy.

Molecular labeling using modern genetically encoded fluorescence proteins or the classic immune-histology is able to provide unique molecular specificity and sensitivity for connection studies. In this issue, Hartmann et al. characterized Cre-recombinase mouse lines that enable selective labeling of cerebrovascular pericytes, which showed a sparse labeling of specific cells for microscopic connectivity studies.

The mesoscale functional connectivity study requires imaging large brain tissue with high spatial resolution to provide a fine connection map, sometimes even with single fiber resolution. Light-sheet microscopy has a 100-year history and has been revisited with another 100-year technique, optical clearing, and radiates vigor and vitality when facing the new challenges from neuroscience. In this issue, the paper by Dodt et al. introduced its development and emphasized the key optical consideration like the objective design for further improvements. In another paper, Müllenbroich et al. introduced the technique details to improve the spatial resolution

C 2015 Society of Photo-Optical Instrumentation Engineers (SPIE) by adopting confocal concept in light-sheet microscopy. This paper further shows how to address the resulting "big data" challenge, another bottle-neck in "omics" studies. To break the limit of optical penetration depth, the idea of serial sectioning has also been revived and combined with light microscopy. Qi et al. introduced the technique details to realize confocal based fluorescence micro-optical sectioning tomography imaging system (fMOST). They applied an acoustooptic deflector, a nonmechanic beam scanner, to provide the high precision and high stability required for long-time continuous microscopic imaging of the brain tissue. Combined with mechanical sectioning and confocal detection, the data enables tracing individual axons.

As emphasized earlier, data analysis models and tools are another bottleneck for connectivity studies. Large-scale imaging and recording over spatial and/or temporal axes with high resolution produced large volumes of data and pose serious challenges on both data storage and processing. In their paper, Lim et al. showed the way to apply network analysis to a regional, functional connectivity map produced by optogenetic stimulation.

Last, standard procedures are important for producing a systematic connection map. The paper by Johnson et al. described the specific connectivity pattern related to horizontal spreads of evoked activity in the rat cortex as such an example. This paper highlights the importance of long-range horizontal connections and the methods to resolve them.

We expect more papers on this and related topics, exploring the neural connectivity with resolution from tens of nanometers to millimeters.

Shaoqun Zeng received his $\mathrm{PhD}$ in optoelectronics at Huazhong University of Science and Technology (HUST), China, in 1996. He is currently a Changjiang professor of biomedical engineering at the Britton Chance Center for Biomedical Photonics, Wuhan National Laboratory for Optoelectronics, HUST. His research focus on developing novel optical principles and systems in particular aims to improve spatio-temporal resolution and throughput to explore the structure and function of neuronal networks.

Timothy H. Murphy obtained his PhD from Johns Hopkins in 1989. $\mathrm{He}$ is a basic scientist interested in applying high-resolution imaging and optogenetic techniques to questions involving stroke in live mice. His lab has evaluated relationships between synaptic structure and brain circuit function during and after ischemia. He is a professor in the Department of Psychiatry and is actively constructing and optimizing instrumentation for in vivo structural and functional brain imaging to investigate mouse models of human disease. 